\title{
Evolution de la stabilité structurale du sol sous l'influence des racines de trèfle (Trifolium pra- tense L.) et de ray-grass (Lolium multiflorum Lmk.). Observations pendant et après culture
}

Joseph E. DUFEY ('), Henri HALEN \& Raymond FRANKART

Université Catholique de Louvain, Science du Sol, 2, place Croix-du-Sud, B 1348 Louvain-la-Neuve, Belgique

RÉSUMÉ

Des cultures, pures et associées, de tréfle violet et de ray-grass italien ont été menées en phytotron durant 15 semaines, sur un sol limoneux. Trois coupes ont eté pratiqueses, après quoi le sol a éte mélangé ct conservì à $25^{\circ} \mathrm{C}$ pendant 12 semaines. On a suivi l'évolution de la stabilité structurale du sol pendant et après la culture, selon la méthode de Hénin. Le poids et la longueur des racines ont été déterminés à chaque coupe. La vitesse de dégagement de $\mathrm{CO}_{2}$ par le sol a été mesurée pendant la période d'incubation.

Le fait le plus notable pendant la période culturale est que le ray-grass augmente nettement le taux d'agrégats stables, alors que le trèfle est quasi inefficient de ce point de vue. Durant la période post-culturale, on observe dans tous les cas un pic immédiat de stabilité des agrégats, suivi d'une lente décroissance de stabilité.

Le taux d'éléments fins diminue de façon monotone pendant la culture et augmente ensuite régulièrement pendant l'incubation. Les 3 traitements culturaux ont des comportements similaires à ce point de vue.

On n'a pas trouvé de relation générale (pour les 3 coupes) entre les mesures de stabilité structurale et les paramètres radiculaires. Une relation simple apparaît toutefois entre la longueur radiculaire en fin de culture ( $3^{\mathrm{c}}$ coupe) et les paramètres de stabilité.

Pendant la période post-culturale, on note un parallélisme entre l'évolution du taux d'agrégats stables et le dégagement de $\mathrm{CO}_{2}$. Cet effet d'origine microbiologique a été interprété par analogie avec l'addition de matière organique fraîche au sol.

Mots clés additionnels : Association végétale, agrégats.

Red clover and Italian rye-grass (pure and in association) were grown in a phytotron for 15 weeks on a loamy soil (table 1). Three cuts were made : afterwards, the soil samples were homogenized in each pot and stored at 25 " $\mathrm{C}$ for 12 weeks. The structural stability of the soil was monitored according to Henin's method, during and after plant growth (fig. 2). Weight and length of roots were measured at each cut (fig. 1). The rate of $\mathrm{CO}_{2}$ evolution from soil samples was measured during the incubation period (fig. 4). During the plant growth period, the percentage of stable aggregates (particles $>200 \mu \mathrm{m}$ ) was clearly improved by rye-grass whereas clover had no significant effect. During soil incubation, a peak of aggregate stability was immediately observed in all cases, followed by a slow decrease of stability. The percentage of fine particles $(<20 \mu \mathrm{m})$ decreased steadily during plant growth and increased during incubation. In this respect, the two plants and their association showed similar behaviour. No general relationship (for the 3 cuts) was found between structural stability and root weight or length. However at the third cut (end of growth period) the structural parameters were well related to root length (fig. 3). During incubation, aggregate stability and $\mathrm{CO}_{2}$ evolution changed in parallel. This effect of microbial origin was interpreted by analogy with the addition of fresh organic matter to soil.

Additional key words : Plant association, aggregates. 


\section{INTRODUCTION}

La stabilité structurale des agrégats du sol est une composante physique essentielle de la fertilité. Pour un sol donné, la stabilité des agrégats est notamment liée à la qualité et à la quantité des matières organiques qu'il contient. Le travail fondamental de MONNIER ( $1965 a$ et $b$ ) sur l'évolution de la stabilité structurale en fonction du type de matière organique retournée au sol a été complété par de nombreux auteurs qui ont bien mis en évidence les effets différentiels des divers constituants organiques du sol: polysaccharides et polyuronides d'origine végétale ou microbienne, substances humiques, substances lipidiques... A titre d'exemples, on peut citer à cet égard les travaux de SWINCER et al. (1969), GREENLAND (1971), MARTIN (1971), GRIFFITHS \& BURNS (1972), JACQUIN (1978).

Une source importante de matières organiques dans le sol est constituée par la racine du végétal. Cet apport peut être direct (décomposition des racines mortes, sécrétions racinaires) ou indirect (stimulation de la prolifération microbienne dans la rhizosphère). En conséquence, comme l'ont montré par exemple Bui HuU TRI \& MONNIER (1973a et $b$ ), TISDAll \& OADES (1979), REID \& GosS (1981), le système radiculaire exerce une action positive sur la stabilité des agrégats du sol. L'origine de cette action n'est toutefois pas uniquement biochimique. TISDALL \& OADES (1979) ont montré que le haut niveau de stabilité observé sous ray-grass était corrélé avec une longueur élevée d'hyphes mycéliens par gramme d'agrégats stables, longueur nettement plus élevée sous ray-grass que sous trèfle. Ces hyphes relient mécaniquement les particules du sol entre elles et, de plus, leur observation au microscope électronique a révélé la présence d'une couche extérieure de matériel amorphe (polysaccharides essentiellement) à laquelle particules d'argiles et micro-agrégats adhèrent fortement. GUCKERT et al. (1975) ont observé des phénomènes analogues à l'interface sol-apex radiculaire.

L'objectif principal du travail présenté ici est de mettre en évidence l'effet différentiel des systèmes radiculaires vivants et morts sur la stabilité des agrégats du sol. Un $2^{e}$ objectif, plus accessoire, est de voir s'il est possible de relier l'effet stabilisateur des racines à des paramètres radiculaires simples, même si l'on sait qu'il s'agit fondamentalement d'une relation complexe.
Cette étude s'insérant dans un projet plus vaste sur l'écologie des associations graminées-légumineuses, le ray-grass et le trèfle ont été choisis comme plantes tests. Ces 2 espèces végétales présentent des systèmes radiculaires très différents tant sur le plan morphologique que sur le plan biochimique (WHITEHEAD et al., 1979 ; STEFFENS, 1984).

\section{MATÉRIEL ET MÉTHODES}

\section{A. Le sol}

Le sol utilisé provient de parcelles expérimentales situées à Gembloux, dans la région limoneuse belge. La plupart des sols de cette région se sont développés dans un limon lœssique déposé au Pléistocène. Il s'agit de sols bruns lessivés (orthic hapludalfs).

Le choix de ce sol a été guidé par la fragilité de la stabilité de ses agrégats; c'est un sol battant très sensible à l'érosion (BOLlinNe \& HANOTIAUX, 1975; BollinNe \& RosseaU, 1978). Cette caractéristique permet donc de détecter aisément tout effet bénéfique sur la stabilité structurale.

Des prélèvements sont opérés dans l'horizon $A_{p}$, entre 5 et $15 \mathrm{~cm}$. Les caractéristiques élémentaires du sol au moment du prélèvement sont données au tableau 1. La teneur en carbone (C) est déterminée par la méthode de WALKLEY et BLACK, à froid. Le pH est mesuré dans une suspension sol/ $\mathrm{KCl} 0,1 \mathrm{M}, 1 / 5$. Les cations échangeables sont extraits par l'Ac- $\mathrm{NH}_{4} 1 \mathrm{M}$ pH 7 en colonnes. Après lavage à l'éthanol, on extrait $\mathrm{NH}_{4}$ par $\mathrm{KCl} 1 \mathrm{M}$ pour la mesure de la capacité d'échange cationique (CEC).

L'analyse minéralogique de la fraction argileuse par diffraction des $\mathrm{RX}$ révèle la présence de kaolinite, d'illite et de traces de smectite en interstratification avec l'illite.

La masse de terre prélevée au champ est passée au tamis de $5 \mathrm{~mm}$ après séchage à l'air. Un $2^{\mathrm{e}}$ tamisage est effectué, sans forcer, à $2 \mathrm{~mm}$. Deux fractions sont donc obtenues : une fraction grossière $2-5 \mathrm{~mm}$ et une fraction fine $0-2 \mathrm{~mm}$, représentant respectivement 45 et $55 \mathrm{p} .100$ en poids de la masse totale.

Des bacs en plastique $(\mathrm{L}=47 \mathrm{~cm}, \mathrm{l}=10 \mathrm{~cm}$, $\mathrm{h}=11 \mathrm{~cm}$ ) sont remplis d'une couche de $1,5 \mathrm{~cm}$ de sable siliceux pur surmontée d'une couche de $7 \mathrm{~cm}$ de terre ; celle-ci est préparée par mélanges successifs de

TABLEAU $\mathrm{I}$.

Caractéristiques du sol.

Soil characteristics.

\begin{tabular}{|c|c|c|c|c|c|c|c|c|c|c|c|}
\hline \multirow[b]{2}{*}{$\mathrm{C} \%$} & \multirow[b]{2}{*}{$\mathrm{pH}_{\mathrm{KCl}}$} & \multicolumn{5}{|c|}{ Complexe d'échange (meq/100 g) } & \multicolumn{5}{|c|}{ Granulométrie $(\%)\left({ }^{1}\right)$} \\
\hline & & $\mathrm{Ca}$ & $\mathrm{Mg}$ & K & $\mathrm{Na}$ & CEC & $0-2 \mu \mathrm{m}$ & $2-20 \mu \mathrm{m}$ & $20-50 \mu \mathrm{m}$ & $50-200 \mu \mathrm{m}$ & $\begin{array}{c}200- \\
2000 \mu \mathrm{m}\end{array}$ \\
\hline 0,63 & 5,50 & 7,72 & 1,01 & 0,54 & 0,14 & 9,04 & 14,4 & 22,5 & 54,9 & 6,65 & 1,55 \\
\hline
\end{tabular}

( ) Selon BOLLINNE et al. (1978). 
petites quantités des 2 fractions de tamisage dans les proportions énoncées ci-dessus. Une technique de ce type est indispensable pour assurer l'homogénéité du substrat en tout point du bac et entre les différents bacs, permettant ainsi d'éviter une certaine stratification granulométrique inhérente à tout échantillonnage d'un matériau constitué de particules ou d'agrégats de tailles très différentes.

\section{B. Les cultures}

Des graines de ray-grass d'Italie (Lolium multiflorum Lamk. var. " Lemtal ») et de trèfle violet (Trifolium pratense L., var. "Violetta ») sont semées en lignes distantes de $2,5 \mathrm{~cm}$, dans le sens de la largeur des bacs. Après la levée, la densité des plants est ajustée à 18 plants. $\mathrm{dm}^{-2}$ par arrachage des plantules excédentaires.

Vingt bacs sont ainsi constitués : 5 de trèfle (T), 5 de ray-grass ( $R$ ), 5 de culture associée (A) (constituée d'un mélange à nombre égal de plants de $T$ et de $R$ ) et 5 bacs témoins $(\mathrm{T})$ sans plante.

Les cultures se déroulent en phytotron à des températures de 18 à $20^{\circ} \mathrm{C}$ le jour $(12 \mathrm{~h})$ et de 13 à $15^{\circ} \mathrm{C}$ la nuit $(12 \mathrm{~h})$. La teneur en eau pondérale du sol est réajustée à 25 p. 100 (soit $2 / 3$ de la capacité au champ) dès que l'évapotranspiration la ramène à 10 p. 100, ce qui donne une fréquence d'arrosage de l'ordre de 1 à 3 j selon l'état de développement des cultures. Les quantités d'eau nécessaires, déterminées par pesée, sont fournies à raison de $2 / 3$ par le dessous des bacs (dans des sous-bacs) et de $1 / 3$ par le dessus, ceci en vue d'assurer une répartition la plus homogène possible des racines dans le substrat. Les bacs subissent une rotation quotidienne dans le phytotron de façon à minimiser les effets éventuels de faibles écarts de conditions climatiques d'un endroit à l'autre.

Trois coupes successives sont pratiquées à $2 \mathrm{~cm}$ audessus de la surface du sol, respectivement 39, 64 et $106 \mathrm{j}$ après le semis. Les parties aériennes sont récoltées et séchées à $60^{\circ} \mathrm{C}$. Au début de chacune de ces périodes de végétation, on ajoute $320 \mathrm{mg} \mathrm{N}-\mathrm{NH}_{4} \mathrm{NO}_{3}$ sur les bacs $\mathrm{R}$ et la moitié de cette dose sur les bacs $\mathrm{A}$.

Après chaque coupe, un bac de chaque type est retenu pour diverses mesures. Les collets des plantes sont coupés au ras de la surface du sol ; la teneur en eau est ajustée à 15 p. 100 ; les bacs sont conservés à $3{ }^{\circ} \mathrm{C}$ pendant $2 \mathrm{j}$ pour assurer une bonne répartition de l'eau ajoutée tout en minimisant les réactions biologiques pendant cette période. Des cylindres en verre (4 à 6 par bac) de $5 \mathrm{~cm}$ de diamètre, affûtés à la base, sont ensuite enfoncés dans le sol. Les bacs sont alors retournés et « démoulés » avec précaution; on retire la couche de sable ainsi qu'une tranche de $1 \mathrm{~cm}$ sur tout le pourtour avec un couteau tranchant. Le contenu des cylindres sert aux mesures des paramètres radiculaires et le sol restant (à l'extérieur des cylindres) est séché à $40{ }^{\circ} \mathrm{C}$ pour les mesures de stabilité structurale.

\section{Les incubations après culture}

Après la $3^{e}$ coupe, les bacs restants sont démoulés comme décrit ci-dessus, sans intrusion préalable de cylindres en verre. Le sol est alors découpé en petits cubes d'environ $2 \mathrm{~cm}$ d'arête. Ceux-ci sont bien mélangés et réintroduits dans les bacs qui sont conservés à $25^{\circ} \mathrm{C}$ pendant 3 mois. La teneur en eau est ajustée régulièrement à 20 p. 100 par vaporisation d'eau déminéralisée.

$\mathrm{Au}$ début de chaque semaine, on prélève environ $200 \mathrm{~g}$ de terre par bac ; ces échantillons sont homogénéisés en vue de prélever des sous-échantillons de $24 \mathrm{~g}$ qui sont introduits dans de petites fioles en verre. Ces fioles sont placées, ouvertes, dans des poudriers de $200 \mathrm{~cm}^{3}$ contenant $20 \mathrm{ml}$ de $\mathrm{KOH} 0,05 \mathrm{M}$. Les poudriers, fermés, sont conservés à $25^{\circ} \mathrm{C}$ durant une semaine. Le titrage du $\mathrm{KOH}$ dans les poudriers avec sol et dans des poudriers témoins sans sol permet de calculer la quantité de $\mathrm{CO}_{2}$ dégagée par l'activité microbienne pendant cette période.

\section{Les paramètres radiculaires}

Les racines sont extraites du sol contenu dans les cylindres en verre de la façon suivante : le sol est plongé dans une solution dispersante de pyrophosphate-Na pendant une nuit. On procède ensuite à une succession d'opérations de lavage à l'eau, de sédimentation des particules minérales (quelques minutes) et de tamisage du surnageant qui contient les racines. Malgré tout le soin que l'on peut apporter à cette opération, la perte de petits fragments de radicelles est inévitable.

La longueur des racines est déterminée par la méthode de NEWMAN (1966) dans une version légèrement modifiée par Rowse \& PHILlLIPS (1974). Elle consiste à compter sous le microscope le nombre d'intersections entre les racines et un réseau de lignes parallèles. Elle est appliquée à 2 aliquotes des racines extraites de chaque cylindre (soit 8 à 12 répétitions par traitement).

Le poids sec des racines est déterminé après séchage à $60{ }^{\circ} \mathrm{C}$.

\section{E. La stabilité structurale}

La stabilité des agrégats est mesurée par la méthode de HENIN et al. (1958) qui s'est avérée très sensible dans le cas des sols limoneux belges (BoLLINNE \& HANOTIAUX, 1975). Brièvement, les étapes successives de la méthode sont : broyage du sol sec, tamisage à $2 \mathrm{~mm}$, humectation à l'eau, agitation, tamisage sous eau avec un appareil de FÉODOROFF (1960). Toutes ces opérations mécaniques sont effectuées dans des conditions standardisées et strictement contrôlées. Les agrégats restés sur le tamis sont séchés à $105^{\circ} \mathrm{C}$ et pesés (soit Ae p. 100 de l'échantillon de départ). Après dispersion à l'hexamétaphosphate- $\mathrm{Na}$, on détermine le taux de sables grossiers ( $>200 \mu \mathrm{m}$, soit $\mathrm{Sg} \mathrm{p.} \mathrm{100).}$ Dans le " filtrat " du tamisage sous eau, on détermine le taux de particules inférieures à $20 \mu \mathrm{m}$ (argile $\mathrm{Ar}$ p. $100+$ limon fin $L$ p. 100) par la méthode de la pipette de Robinson, sans agent dispersant.

L'indice d'instabilité, Is, est alors simplement défini par

$$
\text { Is }=\frac{A r+L}{A e-0,9 S g}=\frac{F}{G}
$$


Le numérateur $F$ (éléments fins) représente donc la tendance à la dispersion et le dénominateur G (agrégats stables) représente la résistance à la désagrégation des fragments initiaux.

\section{RÉSULTATS ET DISCUSSION}

\section{A. Croissance des parties aériennes et racinaires}

La figure 1 présente l'évolution de la croissance des parties aériennes et des racines au cours de la culture. Les traits verticaux représentent 2 fois la valeur de l'écart-type des différentes mesures. La variabilité des paramètres radiculaires est supérieure à celle des parties aériennes. Cela est vraisemblablement lié davantage aux difficultés techniques des mesures qu'à une réalité physique. Cette figure permet de relever diverses particularités des cultures, dont la plupart sont bien connues. L'implantation du trèfle est plus lente que celle du ray-grass de sorte que celui-ci domine d'abord dans l'association (75 p. 100 en $1^{\text {re }}$ coupe) ; le taux de ray-grass régresse ensuite pour atteindre environ 50 p. 100 en $3^{\text {e }}$ coupe. Le rapport pondéral racines/parties aériennes est toujours plus faible chez le trèfle que chez le ray-grass. Les différences morphologiques font que, à poids égal, les racines du ray-
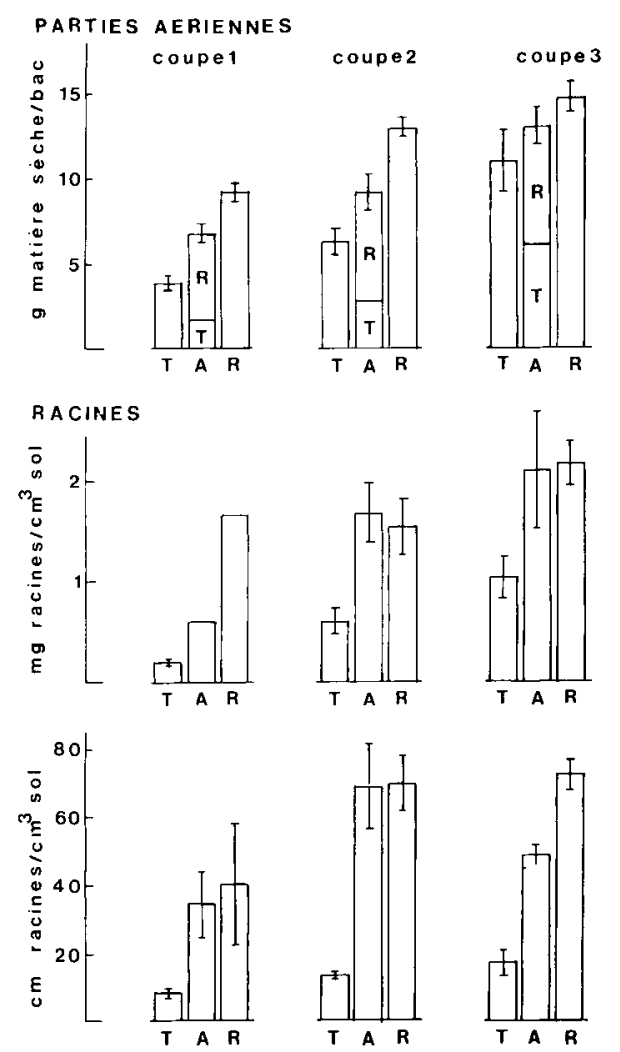

Figure 1

Production de matière sèche et caractéristiques radiculaires des plantes au cours des 3 coupes.

( $T:$ trèfle, $R:$ ray-grass, $A$ : association).

Dry matter yield and root characteristics of the plants after 1, 2, and 3 cuts.

$(T:$ clover, $R:$ rye-grass, $A:$ association $)$. grass développent une longueur supérieure à celles du trèfle, du moins à partir de la $2^{\mathrm{e}}$ coupe ; cela est dû essentiellement au développement du pivot de la racine de trèfle. Dans le mélange d'espèces, les paramètres radiculaires globaux se rapprochent de ceux de la culture pure de ray-grass.

Les données de longueur radiculaire peuvent parâ̂tre très élevées. Elles sont toutefois comparables à celles de la littérature (par ex.: Tisdall \& OAdes, 1979). Exprimées en $\mathrm{km} \cdot \mathrm{m}^{-2}$, les valeurs obtenues pour le trèfle et le ray-grass en $3^{\mathrm{e}}$ coupe sont de 11,4 et 47,4 respectivement.

Sur la figure 2 , on a reporté les valeurs de toutes les mesures de stabilité structurale, durant la période de végétation et durant la période d'incubation. Les écarts-types n'apparaissent pas sur la figure pour des raisons de clarté. Les coefficients de variation (écarttype/moyenne) sont, en moyenne, de 14 p. 100 pour l'indice d'instabilité Is, de 13 p. 100 pour le taux d'agrégats stables $G$ et de 6 p. 100 pour le taux de particules fines $F$.

\section{B. Evolution de la stabilité structurale pendant la cul- ture}

Au cours de la période de végétation, on note une diminution de $F$ et une augmentation de $G$ surtout entre la $1^{\text {re }}$ et la $2^{\mathrm{e}}$ coupe. Le sol témoin sans culture a
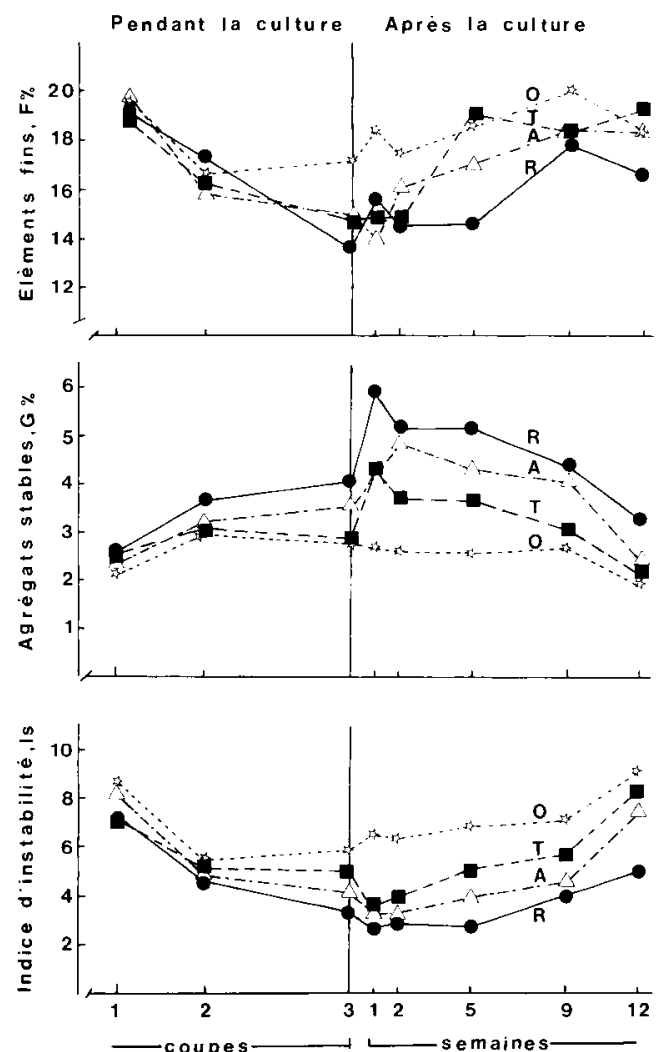

Figure 2

Evolution de la stabilité structurale des sols pendant et après la culture.

- T: Trèfle; clover. $\triangle A:$ Association.

- $R:$ Ray-grass; rye-grass it $O:$ Sol nu; soil without plant. Structural stability of the soil during and after cropping. Top : fine particles $(F, 0-20 \mu \mathrm{m})$; centre : stable aggregates $(G, 0.2-2 \mathrm{~mm})$; bottom : instability index $(I s=F / G)$. 
un comportement parallèle à celui des autres sols de sorte que la plante n'est pas seule responsable des modifications observées. D'autres facteurs tels que les variations d'humidité liées aux cycles d'arrosage et à la vie microbienne non rhizosphérique sont évidemment susceptibles de provoquer une évolution de la stabilité structurale du sol. Le développement de mousses superficielles et d'algues microscopiques sont d'autres causes possibles des variations observées sur le sol nu. Les différences entre cultures se marquent essentiellement sur le taux d'agrégats stables G. A ce point de vue, le sol sous trèfle ne se distingue toutefois pas du sol témoin.

On a tenté de relier les paramètres structuraux aux paramètres radiculaires de la figure 1 . De façon générale, les modifications des 2 types de paramètres sont plus marquées entre les $1^{\text {re }}$ et $2^{\mathrm{e}}$ coupes qu'entre les $2^{\mathrm{e}}$ et $3^{\text {e }}$ coupes. On n'a pas pu toutefois mettre en évidence de relation générale bien précise entre densité pondérale ou longueur de racines et stabilité structurale pour l'ensemble des données. Cependant pour les mesures effectuées en $3^{e}$ coupe, une relation intéressante est apparue entre les paramètres structuraux et la longueur radiculaire (fig. 3).

Il serait toutefois conjectural de voir dans cette liaison une relation de cause à effet. En effet, si, par exemple, on suppose que la stabilisation de la structure est surtout dépendante de la longueur des hyphes mycéliens associés aux racines (TISDALL \& OADES, 1979), il faut encore établir une relation entre longueur d'hyphes et longueur de racines. Si, d'un autre côté, on suppose que la stabilisation structurale est liée à l'activation rhizosphérique microbienne (REID \& Goss, 1981), il faut établir une relation entre excrétion de substrat biodégradable et longueur de racine.

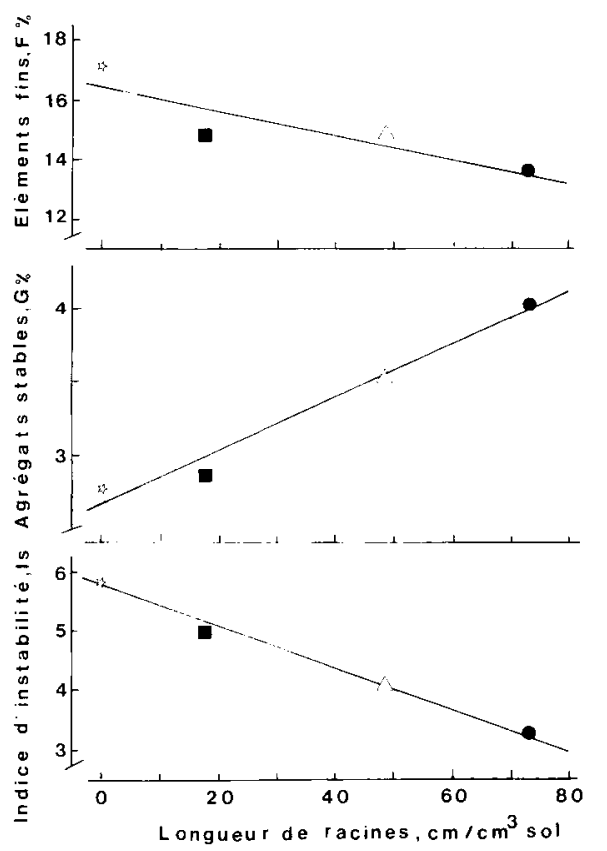

Figure 3

Relation entre la stabilité structurale du sol et la longueur de racines, à la $3^{e}$ coupe.

Relationship between structural stability of the soil and root length at the 3rd cut (see fig. 2 for symbols).
Même si ces hypothèses sont plausibles, ce travail ne permet évidemment pas d'établir la preuve de leur validité, d'autant plus que 2 espèces végétales différentes étaient impliquées.

Il nous paraît cependant raisonnable d'imputer l'effet stabilisateur observé à un facteur directement lié à la présence des racines et non à un début de leur décomposition. En effet, selon TrOUGHTON (1981), la probabilité de trouver des racines mortes après 15 semaines de végétation est très faible. Cet auteur mentionne des temps de vie radiculaire moyens de plusieurs centaines de jours pour différentes espèces herbagères.

\section{Evolution de la stabilité structurale après la cul- ture}

Après la culture, on assiste à une remontée lente du taux d'éléments fins F (fig. 2), jusqu'à atteindre après 9 semaines, des valeurs sensiblement identiques à ce qu'elles étaient au départ.

Le taux d'agrégats stables $G$ augmente fortement dès la $1^{\text {re }}$ semaine, sauf pour le sol témoin. Le trèfle produit également un effet post-cultural très favorable alors qu'il était quasi inefficient pendant la culture. Après un pic initial, on observe une lente décroissance de $(i$, et des valeurs du même ordre de grandeur que celles du départ sont atteintes après 12 semaines d'incubation.

La figure 4 présente l'évolution de la vitesse de dégagement de $\mathrm{CO}_{2}$. Le parallélisme avec l'évolution du taux d'agrégats stables est flagrant. Une production importante de $\mathrm{CO}_{2}$ la ${ }^{\mathrm{re}}$ semaine chez le trèfle et le ray-grass se traduit par un pic de stabilité. Dans

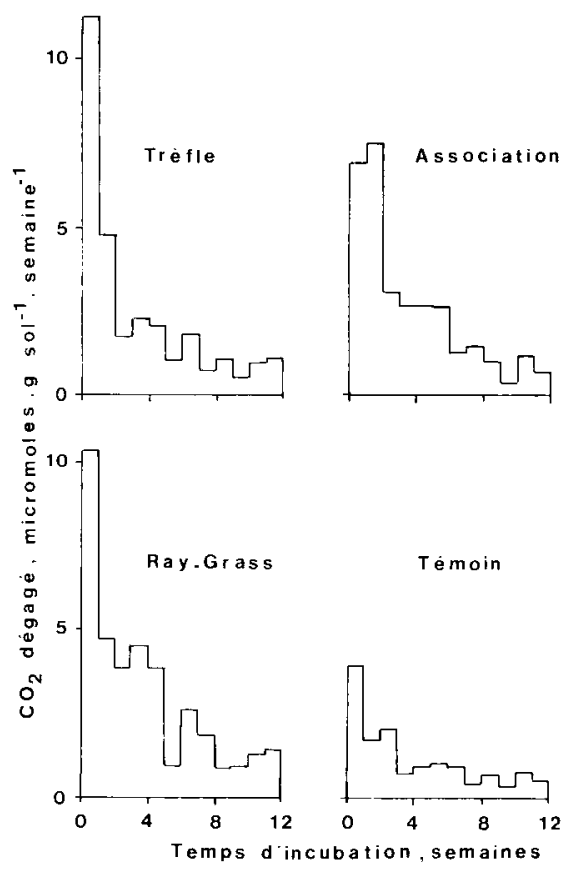

Figure 4

Vitesse de dégagement de $\mathrm{CO}_{2}$ par le sol après les cultures.

Rate of $\mathrm{CO}_{2}$ evolution (tumol.g '. week ') with incubation time (weeks). 
l'association, la production initiale de $\mathrm{CO}_{2}$ est un peu moindre, mais elle se maintient à un niveau élevé pendant 2 semaines. On constate que le maximum de stabilité des agrégats est également atteint après 2 semaines dans ce cas. Il s'agit pourtant de mesures sur des échantillons différents.

La comparaison des figures 2 et 4 indique clairement l'origine biologique de l'effet de l'incorporation de racines mortes sur la stabilité structurale. Des effets similaires sont généralement observés lors de l'addition au sol d'une matière organique fraîche (par ex. : MonNier, 1965 $a$ et $b$; JACQUin, 1978).

On assiste d'abord à une phase d'agrégation intense due à la prolifération explosive des corps microbiens. Ceux-ci interviennent notamment par les filaments fongiques qui relient les particules mécaniquement (DOMMERGUES \& MANGENOT, 1970) et par les cellules microbiennes encapsulées ou productrices de mucus qui engluent les particules (par ex. : JACKSON et al., 1947 ; FOSTER \& ROVIRA, 1976 ; FOSTER, 1978). Les produits du métabolisme microbien sont en grande partie de nature polysaccharidique et présentent un effet agrégatif immédiat très marqué (par ex. : MeHTA et al., 1960 ; MARTIN, 1971). Leur action est toutefois fugace étant donné leur forte biodégradabilité.

Après une décomposition rapide des constituants radiculaires tels que sucres et amidon, le ralentissement de l'activité microbienne correspondrait, en $1^{\text {re }}$ approximation, à la décomposition de la cellulose et des hémicelluloses ainsi que des cellules microbiennes formées en $1^{\text {re }}$ phase. Dans cette $2^{\text {e }}$ phase, les substances responsables de la stabilisation des agrégats seraient les produits transitoires de l'humification.

Une phase de stabilisation durable serait ensuite due à la formation de substances humiques stables. Etant donné la faible quantité de matière organique fraîche retournée au sol dans nos expériences ( 1 à $2 \mathrm{mg}$ de racines. $\mathrm{cm}^{-3}$ ), ainsi que la durée limitée des incubations ( 3 mois), on ne pouvait pas s'attendre ici à mettre clairement cette $3^{\mathrm{e}}$ phase en évidence.

Par rapport au sol témoin, on remarque que le sol$\mathrm{R}$ garde le niveau de stabilité structurale le plus élevé. Cependant, l'accroissement post-cultural du taux d'agrégats stables est du même ordre de grandeur pour les 3 cultures. La masse de racines de trèfle retournée au sol est pourtant 2 fois moindre que celle du ray-grass. Les compositions différentes des racines des 2 espèces en sont l'explication vraisemblable. Une analyse biochimique détaillée est donnée par WHITE-
HEAD et al. (1979). On note un rapport $\mathrm{C} / \mathrm{N}$ de 34 pour des racines de ray-grass et de 22 pour des racines de trèfle. La plus longue durée de la $1^{\text {re }}$ phase de stabilisation dans l'association végétale ( 2 semaines au lieu d'une seule pour les cultures pures) ne semble pas explicable a priori.

\section{CONCLUSION}

Cette étude a permis de bien discerner les effets des systèmes radiculaires vivants (en majeure partie du moins) et morts sur la stabilité structurale du sol. Cette distinction est primordiale puisque, dans le cas du trèfle par exemple, aucun effet réel n'est apparu après 15 semaines de culture, alors qu'un effet positif immédiat s'est manifesté dès que la racine a cessé d'être constituant de la plante pour devenir constituant du sol à part entière.

Dans la pratique, après un certain temps d'implantation du végétal, les 2 effets se manifestent plus ou moins simultanément si le système radiculaire est de type pérenne à renouvellement annuel important. En tout état de cause, la «soudaineté » et la brièveté de l'effet stabilisateur intense observé en post-culture montrent que ce rôle bénéfique des antécédents prairiaux ne peut être valorisé que grâce à une maîtrise extrêmement précise des techniques d'enfouissement et notamment de leur position chronologique par rapport aux phases culturales sensibles, telles que celles de l'implantation des cultures.

En ce qui concerne le $2^{e}$ objectif mentionné dans l'introduction, les résultats sont peu probants. Il apparaît difficile de faire la liaison entre un paramètre radiculaire simple et l'effet stabilisateur. Un protocole expérimental plus spécifique s'avère nécessaire.

Reçu le 8 novembre 1985. Accepté le 9 juin 1986.

\section{REMERCIEMENTS}

Les auteurs remercient le Service de la Science du Sol de la Faculté des Sciences Agronomiques de Gembloux (Dir. Prof. HaNoTIAUX), particulièrement le Dr. Bol.L.INNE pour son aide et ses conseils à divers niveaux de cette étude.

Les auteurs remercient également Mr. P. Populaike (Commission des Sols de Wallonie) pour le tracé des graphiques. 


\section{RÉFÉRENCES BIBLIOGRAPHIQUES}

Bollinne A., Hanotiaux G., 1975. Mesure de la stabilité structurale dans les sols limoneux. Bull. Rech. agron. Gembloux, 10, 247-258.

Bollinne A., Rosseau P., 1978. L'érodibilité des sols de Moyenne et Haute Belgique. Bull. Soc. Géogr. Liège, 14, 127-140.

Bollinne A., Hanotiaux G., Pissart A., 1978. L'érosion en milieu agricole. Pédologie, 28, 233-245.

Bui Huu Tri, Monnier G., 1973a. Etude quantitative de la granulation des sols sous prairies de graminées. I. Paramètres définissant la structure granulaire et leur relation avec la porosité du sol. $A n n$. agron., 24, 401-424.

Bui Huu Tri, Monnier G., $1973 b$. Etude quantitative de la granulation des sols sous prairies de graminées. II. Les paramètres de gra nulation en relation avec la constitution physique du sol, et le système racinaire. Ann. agron., 24, 651-677.

Dommergues Y., Mangenot F., 1970. Ecologie microbienne du sol. Masson \& Cie, Paris, 796 p.

Feodoroff A., 1960. Evaluation de la stabilité structurale d'un sol (indice S). Nouvelles normes d'emploi pour l'appareil à tamiser. Ann. agron., 11, 651-659.

Foster R. C., 1978. Ultramicromorphology of some South Australian soils, 103-109. In Emerson W. W., Bond R. D. \& Dexter A. R. : «Modification of soil structure ». London Wiley, 442 p.

Foster R. C., Rovira A. D., 1976. Ultrastructure of wheat rhizosphere. New Phytol., 76, 343-352.

Greenland D. J., 1971. Interaction between humic and fulvic acids and clays. Soil Sci., 111, 34-41.

Griffiths E., Burns R. G., 1972. Interaction between phenolic substances and microbial polysaccharides in soil aggregation. Plant Soil, 36, 599-612.

Guckert A., Breisch H., Reisinger O., 1975. Interface sol-racine. I. Etude au microscope électronique des relations mucigel-argilemicroorganismes. Soil Biol. Biochem., 7, 241-250.

Henin S., Monnier G., Combeau A., 1958. Méthode pour l'étude de la stabilité structurale des sols. Ann. agron., 1, 73-92.
Jackson M. L.. Mackie W. Z., Pennington R. P.. 1947. Electronmicroscope applications in soil research. Soil Sci. Soc. Am. Proc., $11,57-63$.

Jacquin F., 1978. Influence de la matière organique sur la structure du sol. 11th Int. Cong. Soil Sci., Edmonton, 369-382.

Martin J. P., 1971. Decomposition and binding action of polysaccharides in soil. Soil Biol. Biochem., 3, 33-41.

Mehta N. C., Streuli H., Muller M., Deuel H., 1960. Role of polysaccharides in soil aggregation. J. Sci. Food Agric., 11, 40-47.

Monnier G., 1965a. Action des matières organiques sur la stabilité structurale des sols (Première partie). Ann. agron., 16, 327-400.

Monnier G., 1965 b. Action des matières organiques sur la stabilité structurale des sols (Deuxième partie). Ann. agron., 16, 471-534.

Newman E., 1966. A method of estimating the total length of root in a sample. J. appl. Ecol., 3, 139-145.

Reid J. B., Goss M. J., 1981. Effect of living roots of different plant species on the aggregate stability of two arable soils. J. Soil Sci., 32, 521-541.

Rowse H., Phillips D., 1974. An instrument for estimating the total length of root in a sample. $J$. appl. Ecol., 11, 309-314.

Steffens D., 1984. Root studies and phosphate uptake of rye-grass and red clover under field conditions. $Z$. Pflanzenernaehr. Bodenkd., 147, 85-97.

Swincer G., Oades J., Greenland D. J., 1969. The extraction, characterization and significance of soil polysaccharides. Adv. Agron., $21,195-235$.

Tisdall J. M., Oades J. M., 1979. Stabilization of soil aggregates by the root systems of rye-grass. Aust. J. Soil Res., 17, 429-441.

Troughton A.. 1981. Length of life of grass roots. Grass Forage Sci., 36, 117-120.

Whitehead D. C., Hazel Buchan, Hartley R. D., 1979. Composition and decomposition of roots of rye-grass and red clover. Soil Biol. Biochem., 11, 619-628. 